\title{
Dynamically stable walk control of biped humanoid on uneven and inclined terrain
}

Citation for published version (APA):

Sun, Z., \& Roos, N. (2018). Dynamically stable walk control of biped humanoid on uneven and inclined terrain. Neurocomputing, 280, 111 - 122. https://doi.org/10.1016/j.neucom.2017.08.077

Document status and date:

Published: 06/03/2018

DOI:

10.1016/j.neucom.2017.08.077

Document Version:

Publisher's PDF, also known as Version of record

Document license:

Taverne

Please check the document version of this publication:

- A submitted manuscript is the version of the article upon submission and before peer-review. There can be important differences between the submitted version and the official published version of record.

People interested in the research are advised to contact the author for the final version of the publication, or visit the DOI to the publisher's website.

- The final author version and the galley proof are versions of the publication after peer review.

- The final published version features the final layout of the paper including the volume, issue and page numbers.

Link to publication

\footnotetext{
General rights rights.

- You may freely distribute the URL identifying the publication in the public portal. please follow below link for the End User Agreement:

www.umlib.nl/taverne-license

Take down policy

If you believe that this document breaches copyright please contact us at:

repository@maastrichtuniversity.nl

providing details and we will investigate your claim.
}

Copyright and moral rights for the publications made accessible in the public portal are retained by the authors and/or other copyright owners and it is a condition of accessing publications that users recognise and abide by the legal requirements associated with these

- Users may download and print one copy of any publication from the public portal for the purpose of private study or research.

- You may not further distribute the material or use it for any profit-making activity or commercial gain

If the publication is distributed under the terms of Article $25 \mathrm{fa}$ of the Dutch Copyright Act, indicated by the "Taverne" license above, 


\title{
Dynamically stable walk control of biped humanoid on uneven and inclined terrain
}

\author{
Zhenglong Sun*, Nico Roos \\ Department of Data Science and Knowledge Engineering, Maastricht University, Bouillonstraat 8-10, Maastricht, LH, 6211, Netherlands
}

\section{A R T I C L E I N F O}

\section{Article history:}

Received 20 January 2017

Revised 17 July 2017

Accepted 19 August 2017

Available online 20 November 2017

\section{Keywords:}

Bipedal walk control

Neural network controller

Uneven terrain

\begin{abstract}
A B S T R A C T
This paper contributes to the literature on energy efficient gaits on unknown terrains for humanoid robots, the locomotion system of which has anthropomorphic characteristics. In this work, we firstly present an energy efficient gait for humanoid robots. The main feature of the new gait is the absence of an area of support. The stiffness-free foot can rotate freely around the ankle joint. This feature makes the gait suited for uneven terrains. We then present a group of neural network controllers to regulate the sagittal and lateral motion of the robot's gait in the presence of an unknown terrain. The resulting gait evaluated on an Aldebaran Nao robot, (1) reduces the energy consumption by $41 \%$ on a flat ground compared to the conventional Aldebaran gait, (2) can handle small disruptions caused by an uneven terrain, and (3) looks more like a human gait. A video showing the gait in the simulator is available.
\end{abstract}

(c) 2017 Elsevier B.V. All rights reserved.

\section{Introduction}

Bipedal walking for humanoid robots is one of the most interesting challenges in robotics. In the papers [1-3], we have investigated the possibility of creating a dynamically stable and energy efficient gait without an area of support. Here, the absence of an area of support means that the ankle joint can move freely while the foot is on the ground. In the sagittal direction the robot's Center of Mass (CoM) is falling forward till the foot of the swing leg touches the ground. In the lateral direction, the robot balances above the stance foot in the single support phase, and falls towards the new stance foot in the double support phase. The falling towards the new stance foot is stopped by putting a force on the new stance leg. The resulting gait ${ }^{1}$ was subsequently evaluated on a real Nao robot. The stability of the gait is validated on a flat ground but not on an uneven terrain. Since there is no feedback in the controller, the robot cannot adjust the gait parameters to compensate for the uneven floor. In this paper, we improve the gait's stability on an uneven terrain by introducing feedback in the controller.

Our contributions are as follows: First, we integrate the previous insights to develop a simple new gait with less energy consumption for omni-directional biped walking. This gait exhibits robust dynamically stable behavior on a flat floor. Second, we

\footnotetext{
* Corresponding author.

E-mail address: z.sun@maastrichtuniversity.nl (Z. Sun).

1 A video of the new gait at: https://project.dke.maastrichtuniversity.nl/robotlab/ ?attachment_id=153.
}

demonstrate that feed-forward neural network controllers can be used to enhance the stability control for the locomotion on an uneven terrain. The simple framework opens the door to developing wider sets of bipedal skill, and is applicable to other types of humanoid robots.

The remainder of this paper is organized as follows. In the next section, we will give a brief overview of existing research about kinematics models for humanoid robots, stability criteria and various approaches to obtain energy efficient bipedal walking. Section 3 briefly describes the new gait that we developed and presented in [1]. We used the Inverted Pendulum Model (IPM) to investigate the energy consumption in the sagittal plane. Subsequently, we extended the model to the lateral plane and describe a gait controller with multiple parameters for a 3D fullbody humanoid robot. The controller can achieve a stable gait on a physical robot in the real world after we optimize its parameters through Policy Gradient Reinforcement Learning (PGRL). Section 4 introduces our work on the neural network controller to enhance the gait's stability on an uneven terrain. Section 5 describes the implementation of proposed methods in the simulator and on a Nao robot. The results are demonstrated in this section as well. Section 6 concludes this paper. We provide a brief summary of the results and outline the future research.

\section{Related work}

\subsection{Models of bipedal walking}

Humanoid robots have complex bodies with irregular shape and mass distribution. Therefore, it is advantageous to obtain an 
elemental representation of the robot's dynamics. Ideal features of a model are simplicity, and both a conceptually and mathematically accurate representation of the dynamics of the real system. The main approaches employed to model the kinematics of humanoid robots are based on the Inverted Pendulum Model (IPM) ([4]), which involves a simplification compared to the body of the robot. The IPM represents the whole body of the robot as a point mass located at the center of mass (CoM) of the actual robot. The point mass is linked to the base of the robot by a telescopic massless leg. Restraining the movements of the CoM to a horizontal plane allows to simplify the motion equation of the IPM. The resulting model is known as the Linear Inverted Pendulum Model (LIPM), which [5] proposed to describe humanoid robot locomotion. The LIPM provides an efficient means to represent the kinematic behavior of the robot and it is therefore a popular tool to understand and manipulate the balance of a humanoid robot. With the LIPM and zero moment point (ZMP) stability criteria [6], institutes/companies have successfully built biped robots that can walk with various gaits adapting to different walking situations (e.g. [7-10]).

\subsection{Energy consumption}

The dynamic model is not the only factor to be considered. The energy consumption of a gait is an important issue. Various approaches have been proposed to reduce the energy consumption of a gait. One of these approaches is passive-dynamic walking where the robot's dynamics are designed to enable a robot to walk down slight slopes without control input, except for the gravitational force. The paper of [11] explained this well. Kuo A. et al. [12] believed that passive-dynamic walkers have three primary flaws: (1) they can only walk down slopes, (2) their gaits are restricted by their dynamics, and (3) they are sensitive to perturbations. Realizing these limitations, researchers [13] have sought to improve passive-dynamic walker by adding actuators.

A second approach to obtain energy efficient bipedal walking is through the application of mechanical compliances. In the work of [13] and [14], springs were added across the hip, thigh, knee and ankle simultaneously. Yang T. et al.[15] exploited parallel knee compliance on the robot ERNIE and discussed how soft/stiff springs affect the energy efficiency at different walking speeds. Jerry P. et al.[16] described the implementation of series-elastic actuation on Spring Flamingo (a MIT's planar bipedal walking robot) to enable the control of the ground reaction forces during walking. In the commercial platform used in our experiments, such hardware modifications are not possible.

A third approach to improve the energy-efficiency of bipedal walking is by designing gaits that minimize the energy cost. The most common means of design is to use parametric optimization of the parameters that specify the gait of the robot. For example, Chevallereau et al. [17] used parametric optimization to design fourth degree polynomial functions that give the joint motions over a step as functions of time. Unlike the previous example, in the work of [18] cubic splines connected at points uniformly distributed along the motion time are used to generate complete optimal steps, including a double support phase.

Parametric optimization methods are also implemented to optimize the walking gait on humanoid Nao robots. In the work of [19], the proposed method models the omni-directional motion as the combination of a set of periodic signals. The parameters controlling the characteristics of the signals are encoded into genes and evolutionary learning is used to learn an optimal set of parameters. The Nao humanoid robot is used as the test platform. Abdolmaleki et al. [20] augmented the 3D inverted pendulum with a spring model and use policy search to optimize the parameters of the walking engines on Nao robots. Shahbazi et al. [21] introduced a two-stage learning algorithm for Central Pattern Generator (CPG) of Nao robot's bipedal walking.

\subsection{Stability control on uneven terrains}

A biped robot with a primal walk controller is capable of walking on flat surfaces. However, it has the defect that its stability may not be guaranteed on uneven terrains. A slight irregularity or an undulation of the ground can undermine the balance of the robot. This defect makes the biped robot less practical in real (outdoor) environments where the ideal flat ground is rare.

A number of researchers have proposed solutions for the stability control problem of biped robot on uneven surfaces [22-27]. In the work of Yi [28], these solutions are divided into three categories:

1. Analyze the ground surface using external sensors such as a laser range finder or a camera [29-31]. The robustness of those methods rely on the measurement accuracy of the sensors. Since the position where the sensor is mounted, is usually above the robot's chest, this kind of solutions is not applicable for a wide range of humanoid robots.

2. Use specialized hardware to ensure the stability of walking on an uneven terrain. Sano et al. [24] introduce a new foot with four passive joints, each of which is equipped with a spring and a sensor, to achieve stable biped walking. Wang et al. [32] enable stable dynamic walking on an uneven terrain using a walking model with mechanically compliant ankles. Kang et al. [25] have developed a new biped foot mechanism capable of making a large support polygon on an uneven terrain using three or four spikes.

3. Gain terrain-adaptive skills using a feedback controller [3336]. In these approaches, a feedback controller tunes the gait parameters to realize stable walking on uneven terrains. Yi et al. [28] proposed a method using foot measurements and an on-line learning algorithm, to estimate the surface gradient. This information is used to modify the robot locomotion and control parameters.

Our proposed work uses feedback control. However, it does not estimate the gradient of the slope, but adapts the torque in the knee of the stance leg, and, in the double support phase, also the torque in the knee of the swing leg based on the position of the center of mass (CoM), its speed and its acceleration. Another difference is that our feedback controller does not use a stability criterion such as the zero-moment point (ZPM).

\section{Our gait}

This section briefly describes our new gait presented in [1-3]. We first analyzed the gait without an area of support using an IPM with telescopic legs. Then we designed a controller which implements the gait on a real Nao robot.

\subsection{Kinematics model in sagittal direction}

The IPM with telescopic legs allows the length of the virtual support leg to vary during a step. We proposed the leg-length policy $\delta:\left[-\frac{\pi}{2}, \frac{\pi}{2}\right] \rightarrow[0,1]$ that determines how much the virtual support leg will be shortened as function of the angle between stance leg with vertical axis. The shortening of the stance leg is realized by bending the knee joint, see the right side of Fig. 1 .

To identify the leg-length policy that minimizes the energy consumption of a robot, we make use of the fact that the robot has to bend the knee in order to shorten the leg. The knee torque is the main factor determining the energy consumption [1]. Fig. 3 shows the optimal leg-length policy $\delta(\alpha)$ as a function of the angle $\alpha$ 


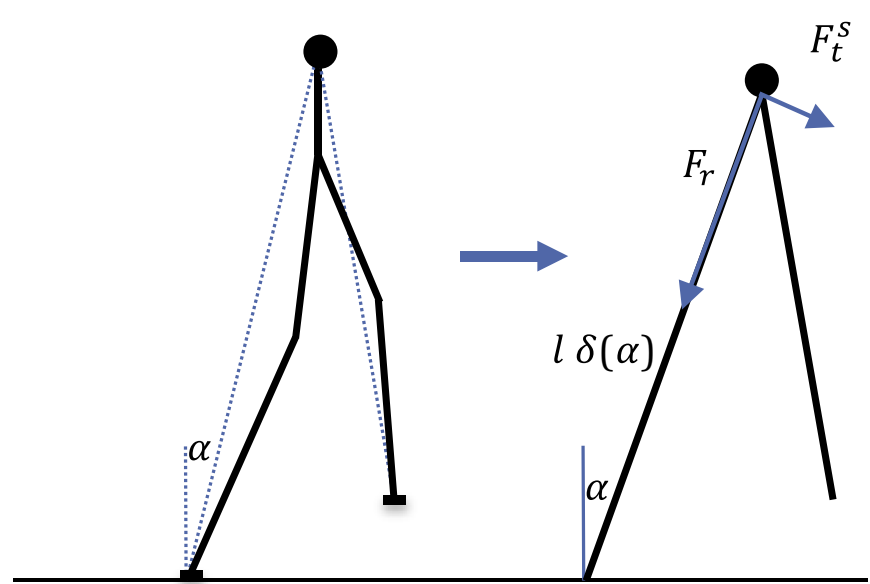

stance leg

Fig. 1. The abstract Inverted Pendulum Model.

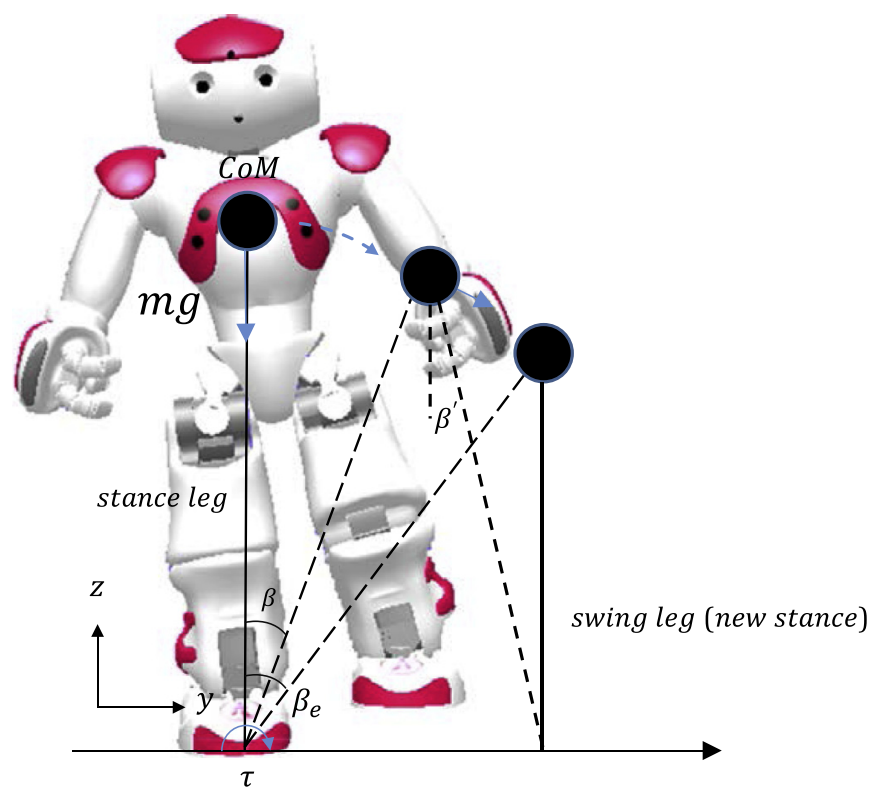

Fig. 2. The CoM lateral movement during double support phase.

$\delta(\alpha)$

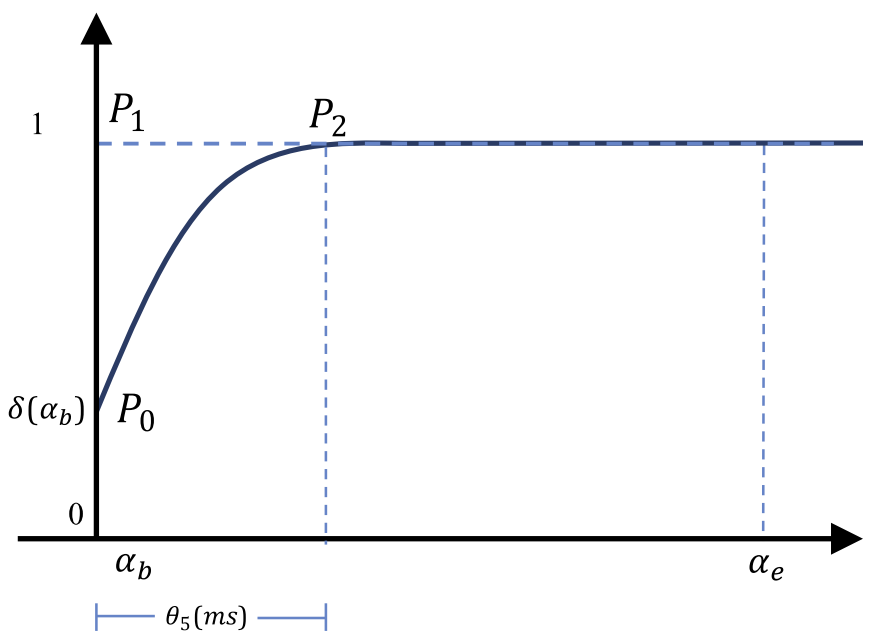

Fig. 3. The optimal leg-length policy.

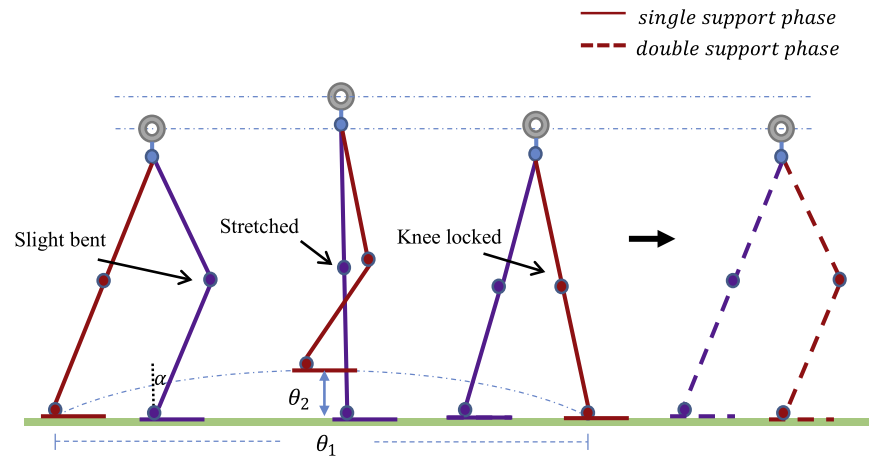

Fig. 4. Kinematic of sagittal motion.

from the beginning till the end of the step that we identified, and Fig. 4 shows the realization using the 5-link model. The detailed information can be found in our previous publication [1].

Since we assumed the absence of an area of support and to further reduce the total energy cost, we set the stiffness on both ankle joints to almost zero. Thus, the stance leg of the robot can freely rotate around ankle joints, and the area of support reduces to a point.

\subsection{Kinematics model in lateral direction}

For a simple forward step, it is insufficient to only consider 2D dynamics in sagittal direction. To address the lateral stability, we designed a lateral controller to regulate the CoM lateral movement during double support phase which is proposed in [3].

At the beginning of the double support phase (DSP) the robot balances in the lateral direction above the stance foot. The angle $\beta$ of the virtual stance leg with the vertical axis is 0 , see Fig. 2 . There is no force on the swing leg when it touches the ground at the beginning of the double support phase. During the double support phase weight of the robot must shift from the stance leg to the swing leg. At the end of the double support phase, the angle $\beta^{\prime}$ of virtual swing leg with the vertical axis must become 0 , see Fig. 2. When the angle of $\beta^{\prime}$ becomes 0 the single leg must carry the full weight of the robot, the robot balances above the swing foot, and the double support phase ends.

We use the upper body tilt to initiate the lateral movement of the CoM towards the swing foot. Next, we use a force generated by the swing leg to stop the movement when CoM is balanced above the swing foot, which then becomes the new stance foot. The force generated by the swing leg is described by a force policy. In order to smooth the CoM transition trajectory, we determine the shape of the force policy by means of Quadratic Bezier curve which introduces the quadratic Bezier point $\theta_{7}$, see Fig. 5. The quadratic Bezier point $\theta_{7}$ is one of the controller parameters discussed in next subsection.

\subsection{Controller design}

Our gait has been implemented on a Nao robot by defining a parametrized controller and then searching for parameter values that yield appropriate control behavior. Policy search $[37,38]$ is a well-known method that has been applied successfully for searching in high-dimensional parameter spaces. This subsection presents the parameters of a gait controller that realizes the leg-length policy described in Section 3.1 and the force policy described in Section 3.2. We identified 9 parameters that are essential in controlling a dynamic gait:

- Step Length $\left(\theta_{1}\right)$ : Defines the distance which Nao moves in a singe step (sagittal). 


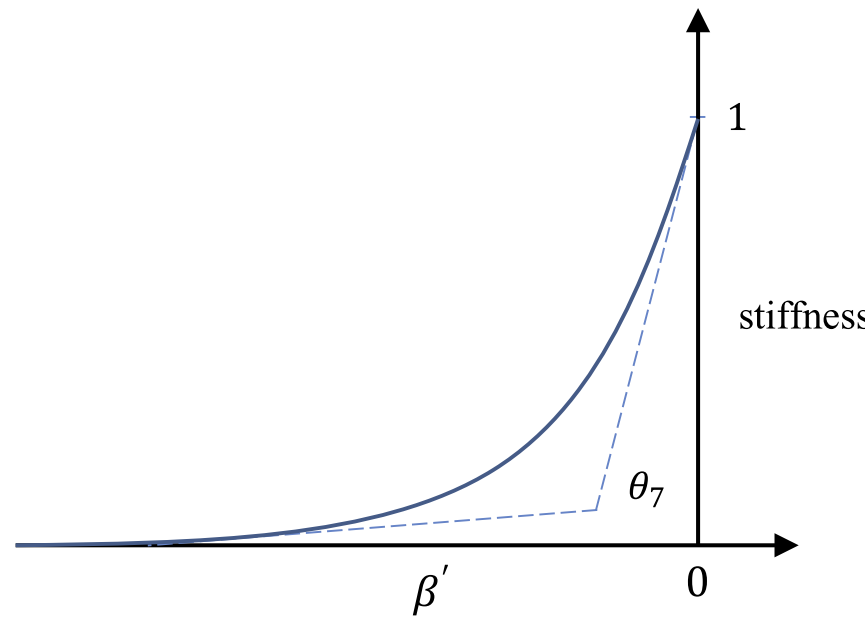

Fig. 5. Stiffness over angle $\beta^{\prime}$ by Quadratic Bezier curves.

- Step Height $\left(\theta_{2}\right)$ : Defines the maximal altitude between ground and lifting feet. A high step height requires a faster movement of the swing leg, which may cause instabilities. A low step height increases the possibility of tripping and limits the step length.

- Knee Bending $\left(\theta_{3}\right)$ : Defines the bending angle of the stance leg (front support leg in Fig. 4) at the beginning of single support phase (SSP). $\delta\left(\alpha_{b}\right)$ in Fig. 3 is represented by the angle $\theta_{3} . \delta\left(\alpha_{b}\right)=1$ corresponds to the knee angle: $180^{\circ}$ (the fully stretched leg), and $\delta\left(\alpha_{b}\right) \approx 0.53$ corresponds to the knee angle: $0^{\circ}$ (the completely bend leg). The parameter $\theta_{3}$ determines the sagittal velocity and the energy cost.

- Step Time $\left(\theta_{4}\right)$ : Defines how long a single step lasts. This parameter determines the sagittal walking velocity.

- Stretch Time $\left(\theta_{5}\right)$ : Defines how long it takes for the stance leg to stretch from $\delta\left(\alpha_{b}\right)$ (angle of bent knee) to its full length, see Fig. 3.

- Torso Pitch Inclination $\left(\theta_{6}\right)$ : Defines the maximum angle that torso leans in sagittal direction at the beginning of the first step. If positive, it will move the center of mass (CoM) in sagittal direction. If it is set not appropriate, a fall will occur. In our experiments, the inclination lasts for $200 \mathrm{~ms}$.

- Quadratic Bezier point $\left(\theta_{7}\right)$ : Defines the magnitude of middle points in Quadratic Bezier Curves, which determines the force policy of the swing leg (introduced in Section: 3 ).

- Torso Roll Inclination $\left(\theta_{8}\right)$ : Defines the maximum angle that torso leans in lateral direction. If positive, it will move the center of mass (CoM) towards the swing leg in lateral plane as discussed in Subsection: 3.2. In our experiments, the inclination lasts for $50 \mathrm{~ms}$.

- Ratio of single support duration $\left(\theta_{9}\right)$ : Defines how long the single support phase lasts in one single step. The single support phase duration equals this parameter times $\theta_{4}$ (the step time).

All parameters except $\theta_{1}$ (the step length) will be optimized in the experiments. We do not consider the step length for optimization because we need step length to be variable when the velocity is changing. We manually set a different walking velocity $v$ in each experiment and determined the optimal Step Time $\theta_{4}$. The corresponding step length is given by: $\theta_{1}=v \theta_{4}$.

\subsection{Learning optimal parameters}

We use a policy gradient reinforcement learning method [39] to search the set of possible parameters with the goal of finding the stable and low energy cost walk. In order to generate a gait that is
Table 1

Learned parameters set $P$.

\begin{tabular}{lll}
\hline Parameter & $\epsilon$ & Learned value \\
\hline Step length $\left(\theta_{1}\right)$ & 0.1 & $3.9(\mathrm{~cm})$ \\
Step height $\left(\theta_{2}\right)$ & 0.02 & $3.24(\mathrm{~cm})$ \\
Knee bending $\left(\theta_{3}\right)$ & 0.1 & $14.2\left(^{\circ}\right)$ \\
Step time $\left(\theta_{4}\right)$ & 25 & $650(\mathrm{~ms})$ \\
Stretch time $\left(\theta_{5}\right)$ & 25 & $78(\mathrm{~ms})$ \\
Torso pitch inclination $\left(\theta_{6}\right)$ & 0.1 & $8.9\left(^{\circ}\right)$ \\
Quadratic Bezier point $\left(\theta_{7}\right)$ & 0.1 & $\left(0.9^{*}\right.$ double support phase time, 0.2$)$ \\
Torso roll inclination $\left(\theta_{8}\right)$ & 0.1 & $6.5\left(^{\circ}\right)$ \\
Ratio $\left(\theta_{9}\right)$ & 0.1 & 0.8 \\
Velocity & & $6(\mathrm{~cm} / \mathrm{s})$ \\
\hline
\end{tabular}

energy efficient and stable, we considered a fitness function based on the total energy cost and the stability over a certain distance of forward walk. The energy cost determines $30 \%$ of the fitness function value and the distance which the robot walks without falling determines $70 \%$ of the fitness function value [1].

To generate the optimal gait parameters and validate the gait's performance, we uploaded the controller of our proposed gait together with an implementation of the policy gradient algorithm into the Webots simulator. We used a relatively elementary handtune gait as a starting policy for the policy gradient algorithm. Each new policy was evaluated by letting a robot walk at a constant distance of 0.75 meters. During the walking, the energy consumption and stability were determined. The policy gradient algorithm converges to a parameters set $P$ shown in Table 1.

The algorithm presented here converges to a local optimum. In order to investigate whether the results could be a global optimum, we repeated the learning experiment 500 times, each time starting from a randomly generated parameter vector $x^{\pi}$ with the same velocity. The results of the experiments indicate that the local optimum we have in Table 1 is probably the global optimum. Therefore, the parameters set $P$ most likely results in the most energy efficient gait. The accompanying video material ${ }^{2}$ shows the Nao robot walking on flat ground with our proposed gait controller at a speed of $6 \mathrm{~cm} / \mathrm{s}$. We also compare the new gait on the flat ground with the standard gait that Aldebaran supplies with the Nao. The energy consumption of the new gait is $41 \%$ less than the Aldebaran gait on a real Nao robot.

\section{Analysis of gait on uneven terrain}

\subsection{Overview of system}

The new gait proposed in [1] uses a controller that generates the joint angles for the knee joint and the hip joints of the stance lag using a leg-length policy, and realizes the force policy described by a Bezier curve for the swing leg by controlling the stiffness of knee joint. Though the results show the effectiveness of using a mixture of a leg length policy, a force policy and a policy gradient reinforcement learning method to obtain a bipedal gait which is specialized in the aspect of energy efficiency, the gait has its own defect that the stability can not be guaranteed when external disturbances occur. This means any disturbance such as a push or stepping on uneven terrain may jeopardize its balance because the ankle stiffness is set to almost zero. For example, if the robot is standing on a slope or stepping on a bump, its feet are not on the same altitude. This may cause the CoM to undershoot or overshoot the balance position when switching the stance leg. To enhance the walking stability especially on complex terrains, we further extend the controller.

\footnotetext{
2 https://project.dke.maastrichtuniversity.nl/robotlab/?attachment_id=153.
} 

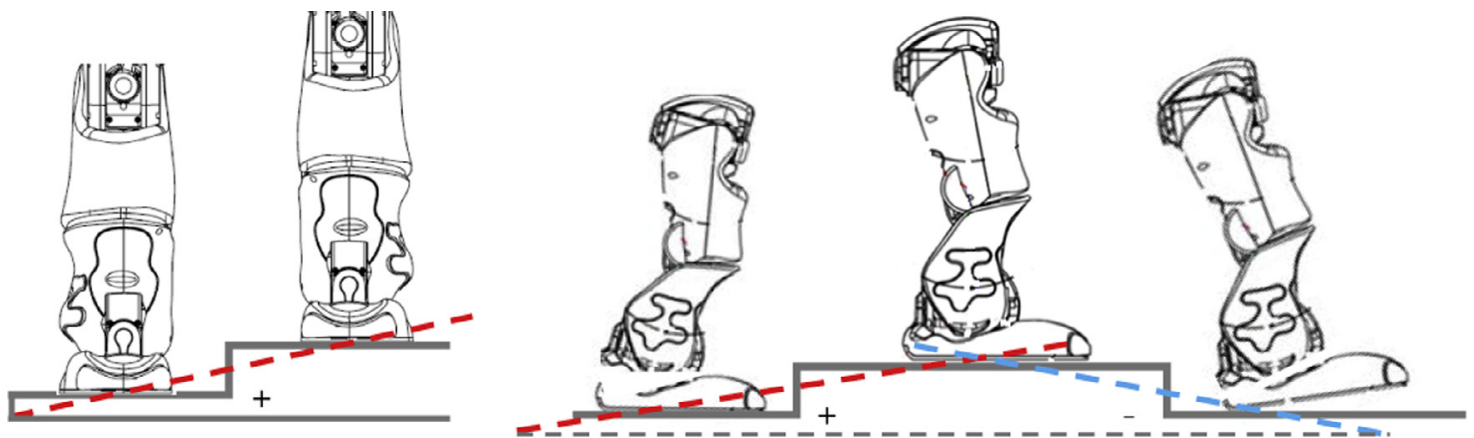

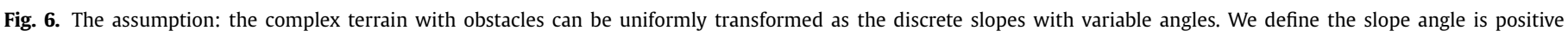
if left foot is landed higher than right foot on lateral plane or new support foot is landed higher than rear foot on sagittal plane and vice versa.

In order to make the problem tractable, we made a simplifying assumption. Since the altitude of robot's two feet can be different when walking on an uneven terrain, each step of the walk on an uneven floor can be viewed as a step on a (virtual) slope. The angle of the slope is determined by the relative elevation of both feet when they are firmly placed on the ground. Therefore, the complex terrain with obstacles, including bumps, pits and slopes, can be uniformly transformed to slopes with variable angles. To solve the balancing problem on an uneven terrain, the gait controller should adapt the gait parameters to the various slopes.

We implemented three neural networks to control and improve the robot's stability on unknown slopes. An overview of the system is shown in Fig. 7, which illustrates two loops that each corresponds to a different step phase. In the following description, we review its operation of the gait control on an uneven terrain.

The upper loop controls the leg length policy in single support phase. During each step, the neural network determines leg length and sent this value to the gait controller. The individual joint torques on the support leg are computed by low-level control structures.

The bottom loop operates during the double support phase. The touch-down of the swing foot marks the beginning of the double support phase, and from this moment the neural network determines the proper stiffness of the swing leg's knee joint.

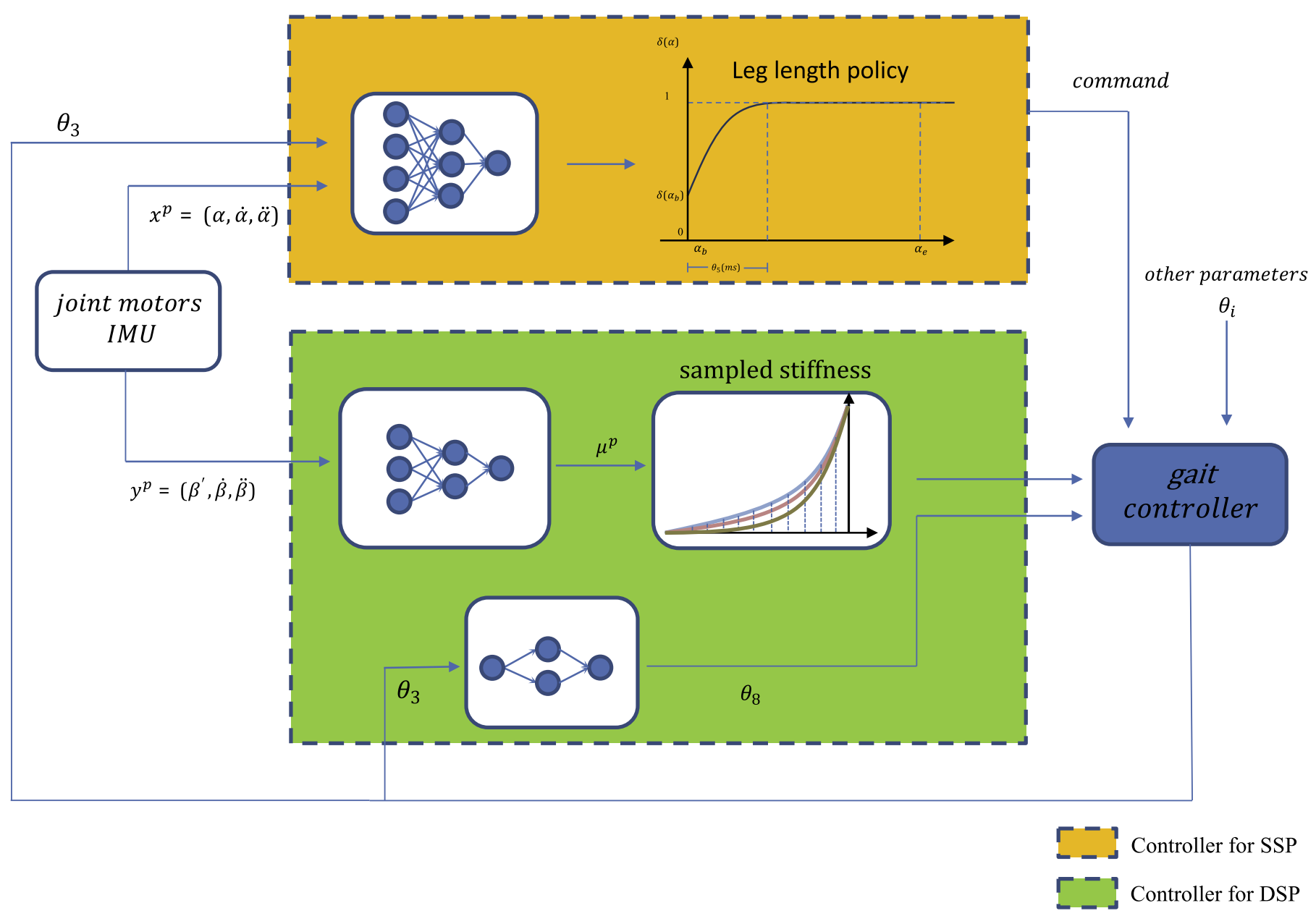

Fig. 7. System overview of the controller in single and double support phase. 
Table 2

Learned control parameters relevant for the single support phase on different slopes in sagittal direction

\begin{tabular}{llllllll}
\hline$p$ (slope angle) & $\mathrm{H}(\mathrm{cm})$ & $\theta_{2}$ & $\theta_{3}$ & $\theta_{4}(\mathrm{~ms})$ & $\theta_{5}(\mathrm{~ms})$ & $\theta_{6}$ & $\theta_{9}$ \\
\hline$-10^{\circ}$ & -0.68 & 3.24 & $8.5^{\circ}$ & 650 & 64 & $0^{\circ}$ & 0.8 \\
$-7^{\circ}$ & -0.48 & 3.24 & $9.2^{\circ}$ & 650 & 68 & $1.4^{\circ}$ & 0.8 \\
$-5^{\circ}$ & -0.34 & 3.24 & $11.7^{\circ}$ & 650 & 70 & $4.1^{\circ}$ & 0.8 \\
$-3^{\circ}$ & -0.20 & 3.24 & $13.7^{\circ}$ & 650 & 75 & $5.7^{\circ}$ & 0.8 \\
$0^{\circ}$ & 0.00 & 3.24 & $14.2^{\circ}$ & 650 & 78 & $8.9^{\circ}$ & 0.8 \\
$3^{\circ}$ & 0.20 & 3.24 & $14.4^{\circ}$ & 650 & 76 & $10.2^{\circ}$ & 0.8 \\
$5^{\circ}$ & 0.34 & 3.24 & $15.2^{\circ}$ & 650 & 75 & $13.5^{\circ}$ & 0.8 \\
$7^{\circ}$ & 0.48 & 3.24 & $15.7^{\circ}$ & 650 & 74 & $14.9^{\circ}$ & 0.8 \\
$10^{\circ}$ & 0.68 & 3.24 & $16.1^{\circ}$ & 650 & 72 & $16.3^{\circ}$ & 0.8 \\
\hline
\end{tabular}

\subsection{Controller design}

Our robot is an Aldebaran H25 Nao (version 5). It is $574 \mathrm{~mm}$ tall and has a mass of $5.4 \mathrm{~kg}$. The pelvis is designated as the root link and each link is connected to its parent link with a revolute joint, yielding a total of 25 internal degrees of freedom. The motion of the joints are driven by the application of internal joint torques and is simulated in the Webots simulator.

Analysis of single support phase. A total of 8 control parameters serve to define the walking gaits. These include the specification of the step height, the target joint angles for the knee and the torso inclination, as applied in the single support phase. Additionally, the parameters about time control of knee stretching and single support phase duration are also part of the parameter set. Therefore, when designing a controller that can handle disturbances influencing the sagittal stability, we determine the proper control parameters which guarantees the robot's stable walk on a uneven terrain in the sagittal direction. Moreover, as we mentioned in this section, the robot's single step on the complex terrain with obstacles is assumed to be one on a (virtual) slope with a certain angle. So when the robot makes one step on the ground, according to the sign of height difference of both feet, we can categorize the terrain where robot's feet are placed into two types: the (virtual) uphill or downhill.

Without the predefined information of terrain, the only available information the robot has is (1) the angle $\alpha$ of the CoM w.r.t the stance foot, (2) the angular velocity $\dot{\alpha}$ of the rotation of the CoM around the ankle of the stance foot, and (3) the sagittal acceleration measured by the Inertial Measurement Unit (IMU). The latter value approximates the angular acceleration $\ddot{\alpha}$ of the CoM w.r.t. the stance foot. We choose to use these variables as inputs of the controller to directly determine the trajectory of leg-length policy.

We designed a series of experiments in the simulator Webots to obtain the optimal control parameters. In the experiments, the same reinforcement learning method as used in Section 3.4, is used to find the proper control parameters that guarantee a stable walking gait on different slopes. The experiments required the robot to walk on slopes where tilt angles varies from -0.17 to 0.17 (rad) in robot's sagittal plane. We let the robot walk with a fixed step length $\left(\theta_{1}=3.9 \mathrm{~cm}\right)$ and execute the reinforcement learning to get the proper controller parameters that ensure the robot's stability. Each new policy was evaluated by letting a Nao robot move for $5 \mathrm{~s}$. The fitness function of each policy will get high score if the robot keeps stable. Otherwise, the function gets a penalized score. After the result of reinforcement learning converged while the robot's movement become stable, the control parameters are recorded.

Table 2 shows the control parameters of the gait on the different slopes and the corresponding feet height difference $H=$ $\theta_{1} \sin (p)$ due to the slope angle $p$. As the slope angle increases from negative to positive, the parameters $\theta_{2}$ (Step Height), $\theta_{4}$ (Step

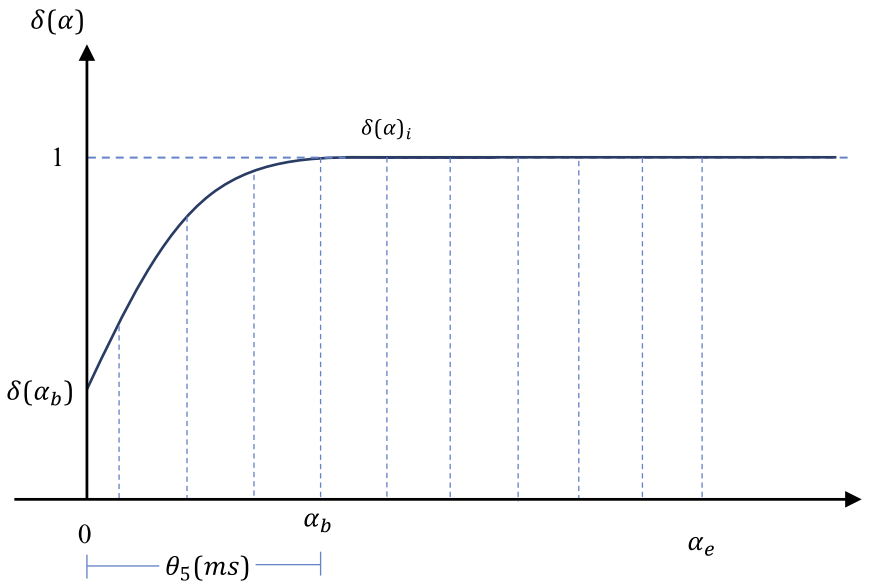

Fig. 8. Sampled leg-length policy.

Time) and $\theta_{9}$ (Ratio) remain constant, while $H$ (feet height difference), $\theta_{3}$ (Knee Bending), $\theta_{5}$ (Stretch Time) and $\theta_{6}$ (Torso Pitch Inclination) depend on the slope angle. Since these control parameters regulate the locomotion in singe support phase, we designed the sagittal controller to adjust those variable parameters to maintain the gait's stability on unknown slopes. Note that, the parameter $\theta_{3}$ (Knee Bending) solely depends on the relative elevation between stance and swing leg, and its value can be determined at end of the double support phase of the previous step.

Sagittal Controller. The main task of sagittal controller is to generate a leg-length policy that is adaptive to unknown slopes. As shown in Fig. 3, the leg length policy is determined by two factors: $\theta_{3}$ and $\theta_{5} \cdot \theta_{3}$ (Knee Bending) (or $\delta\left(\alpha_{b}\right)$ which represents it in percentage) is the knee joint angle at the beginning of single support phase. The parameter $\theta_{3}$ (Knee Bending) solely depends on the relative elevation between stance and swing leg, and its value has to be updated when a new step begins.

In the previous work, we regulate the trajectory of the leg length policy with Quadratic Bezier Curve in the following equation:

$B(t)=\left(\theta_{5}-t\right)\left[\left(\theta_{5}-t\right) P_{0}+t P_{1}\right]+t\left[\left(\theta_{5}-t\right) P_{1}+t P_{2}\right]$

where $0 \leq t \geq \theta_{5}, \quad P_{0}=\left(0, \delta\left(\alpha_{b}\right)\right), \quad P_{1}=(0,1), \quad P_{2}=\left(\theta_{5}, 1\right) \quad$ (see Fig. 3). Given $\theta_{3}$ (Knee Bending) and $\theta_{5}$ (Stretch Time), this equation can generate a smooth trajectory from 0 to $\theta_{5}$ in $x$ axis and from $\delta\left(\alpha_{b}\right)$ to 1 in $y$ axis. This method is feasible for the gait on the flat ground, but it is not a robust method for the gait on the uneven terrain. Without the predefined information of terrain, the parameter $\theta_{5}$ (Stretch Time) of each step are hard to determine. Therefore, we decided to use a trained neural network to directly generate the trajectory of leg-length policy (see Fig. 8) that is adaptive to unknown slope using $\alpha, \dot{\alpha}, \ddot{\alpha}$ and $\theta_{3}$ as inputs.

Analysis of Double Support Phase. When designing the controller that can handle disturbances during the double support phase, we determined the control parameters of the gait which guarantee the robot's side-way stability on an uneven terrain. (Note that, in all the experiments of this section, the length of step is fixed, so the relevant control parameters $\theta_{1}$ is excluded from the optimization.) Since left and right foot are at different heights, the control parameters for the left and right leg might be different. Therefore, the control parameters are split into two parameter sets for the left leg $\theta_{i}^{L}$ and the right leg $\theta_{i}^{R}$, respectively. Next we addressed how to adapt the control parameters.

The robot does not know that it walks on a slope or about other disturbances. The only available information it has is (1) the 
Table 3

Learned control parameters relevant for the double support phase on different slopes in lateral direction.

\begin{tabular}{llllllllll}
\hline$p$ & $\mathrm{H}(\mathrm{cm})$ & $\theta_{2}^{L}(\mathrm{~cm})$ & $\theta_{3}^{L}$ & $\theta_{4}^{L}(\mathrm{~ms})$ & $\theta_{5}^{L}(\mathrm{~ms})$ & $\theta_{6}^{L}$ & $\theta_{7}^{L}$ & $\theta_{8}^{L}$ & $\theta_{9}^{L}$ \\
\hline $0^{\circ}$ & 0.00 & 3.24 & $14.2^{\circ}$ & 650 & 78 & $8.9^{\circ}$ & $(0.900 .20)$ & $6.5^{\circ}$ & 0.8 \\
$1^{\circ}$ & 0.17 & 3.24 & $14.5^{\circ}$ & 650 & 75 & $8.9^{\circ}$ & $(0.860 .16)$ & $5.5^{\circ}$ & 0.8 \\
$2^{\circ}$ & 0.33 & 3.24 & $15.3^{\circ}$ & 650 & 76 & $8.9^{\circ}$ & $(0.840 .14)$ & $3.5^{\circ}$ & 0.8 \\
$3^{\circ}$ & 0.50 & 3.24 & $15.5^{\circ}$ & 650 & 74 & $8.9^{\circ}$ & $(0.800 .12)$ & $2.0^{\circ}$ & 0.8 \\
$4^{\circ}$ & 0.66 & 3.24 & $16.1^{\circ}$ & 650 & 72 & $8.9^{\circ}$ & $(0.780 .12)$ & $1.5^{\circ}$ & 0.8 \\
$5^{\circ}$ & 0.83 & 3.24 & $16.4^{\circ}$ & 650 & 70 & $8.9^{\circ}$ & $(0.760 .12)$ & $1.5^{\circ}$ & 0.8 \\
$p$ & $\mathrm{H}(\mathrm{cm})$ & $\theta_{2}^{R}(\mathrm{~cm})$ & $\theta_{3}^{R}$ & $\theta_{4}^{R}(\mathrm{~ms})$ & $\theta_{5}^{R}(\mathrm{~ms})$ & $\theta_{6}^{R}$ & $\theta_{7}^{R}$ & $\theta_{8}^{R}$ & $\theta_{9}^{R}$ \\
$0^{\circ}$ & 0.00 & 3.24 & $14.2^{\circ}$ & 650 & 78 & $8.9^{\circ}$ & $(0.900 .20)$ & $6.5^{\circ}$ & 0.8 \\
$1^{\circ}$ & 0.17 & 3.24 & $13.5^{\circ}$ & 650 & 74 & $8.9^{\circ}$ & $(0.900 .22)$ & $6.5^{\circ}$ & 0.8 \\
$2^{\circ}$ & 0.33 & 3.24 & $11.7^{\circ}$ & 650 & 70 & $8.9^{\circ}$ & $(0.910 .26)$ & $7.5^{\circ}$ & 0.8 \\
$3^{\circ}$ & 0.50 & 3.24 & $9.6^{\circ}$ & 650 & 68 & $8.9^{\circ}$ & $(0.920 .28)$ & $8.5^{\circ}$ & 0.8 \\
$4^{\circ}$ & 0.66 & 3.24 & $8.5^{\circ}$ & 650 & 65 & $8.9^{\circ}$ & $(0.920 .29)$ & $9.5^{\circ}$ & 0.8 \\
$5^{\circ}$ & 0.83 & 3.24 & $8.2^{\circ}$ & 650 & 65 & $8.9^{\circ}$ & $(0.940 .29)$ & $9.5^{\circ}$ & 0.8 \\
\hline
\end{tabular}

angle $\beta^{\prime}$ of the CoM w.r.t the swing foot (see Fig. 2), (2) the angular speed $\dot{\beta}$ of the rotation of the CoM around the ankle of the stance leg, and (3) the lateral acceleration measured by the Inertia Measurement Unit (IMU). The latter value approximates the angular acceleration $\ddot{\beta}$. We chose to use these three parameters as inputs for the controller that can adapt the gait parameters.

We designed the same experiments as we did for sagittal controller to obtain the optimal control parameters. In the experiments, the same policy reinforcement learning method as used in Section 3.4, is used to find the proper control parameters that guarantee a stable walking gait on different slopes. The experiments require the robot to walk on various slopes where tilt angles varies from -0.09 to 0.09 (rad) in robot's lateral plane. Since in the lateral plane, slopes with negative tilt angles are opposite to these with positive angles, the results of the left leg on slopes with negative angles is identical to the results of the right leg on slopes with positive angles and vice versa. Therefore, we decide to run the experiments on slopes where tilt angles varies only from 0 to 0.09 (rad) in robot's lateral plane (see Fig. 6). We kept the robot walking on the slope and ran the reinforcement learning method to get the controller parameters that ensure the robot's stability. Each new policy was evaluated by letting a Nao robot move for $5 \mathrm{~s}$. The fitness function of each policy will get high score if the robot keeps stable. Otherwise, the function gets a penalized score. After the result of reinforcement learning converged while the robot's movement become stable, the control parameters are recorded.

Table 3 shows the control parameters of the gait on the different slopes and corresponding feet height difference $H=D \sin (p)$ due to the slope angle $p$. Here, $D$ denotes the distance between both feet in the lateral direction, which is $9.5 \mathrm{~cm}$ in our experiments. As the slope angle increases from $0^{\circ}$ to $5^{\circ}$, the parameters $\theta_{2}, \theta_{4}, \theta_{6}$ and $\theta_{9}$ keep constant, while $H, \theta_{3}, \theta_{5}, \theta_{7}$ and $\theta_{8}$ depend on the slope angle. The parameters $\theta_{3}$ and $\theta_{5}$ overlap with the parameters controlled by the sagittal controllers. Tables 2 and 3 show that the same height difference $H$ results in the same values for the parameters $\theta_{3}$ and $\theta_{5}$. Therefore, the lateral controller only needs to adapt $\theta_{7}$ and $\theta_{8}$. Moreover, the sagittal and the lateral controller can operate independently. We designed the controller for double support phase to adjust the parameters $\theta_{7}$ and $\theta_{8}$ to maintain the gait's stability on unknown slopes.

Lateral Controller. To maintain the gait's stability on unknown slopes, the parameters $\theta_{7}$ and $\theta_{8}$ must be controlled. Note that, the parameter $\theta_{3}$ solely depends on the relative elevation between the stance and the swing leg, and its value can be determined at end of the double support phase where $\beta^{\prime}$ should become zero (shown in Fig. 2). Since $\theta_{3}$ encodes the information about the slope angle, its value can be used to set the value of parameters $\theta_{8}$. Therefore, we use a neural network which takes the input the value of $\theta_{3}$ to output the values of $\theta_{8}$.

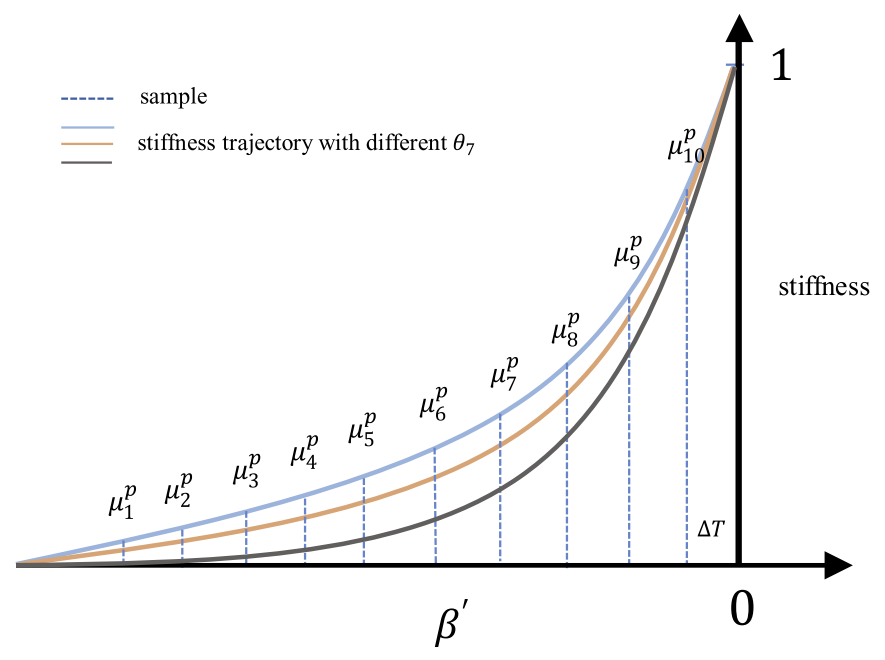

Fig. 9. Sampled stiffness over angle $\beta^{\prime}$.

Besides, the parameter $\theta_{7}$, relevant to stiffness trajectory, needs to be controlled as well. However, $\theta_{7}$ cannot be adapted by a neural network that uses $\left(\beta^{\prime}, \dot{\beta}, \ddot{\beta}\right)$ as inputs. At the end of the double support phase, $\left(\beta^{\prime}, \dot{\beta}, \ddot{\beta}\right)$ must become equal to $(0,0,0)$ for every $\theta_{7}$ value. So, in the neighborhood of $(0,0,0)$ the correct $\theta_{7}$ value is not well defined. Therefore, instead of $\theta_{7}$, the trajectory of stiffness will be directly determined by a neural network controller. The sampled stiffness values from the trajectories defined by different values of $\theta_{7}$ can be seen in Fig 9.

\section{Controller implementation and evaluation}

We implemented three neural networks to control the robot's stability by adjusting the controller parameters to unknown slope. The back-propagation method has been applied for training multilayer feed-forward networks, see Fig. 10. With the trained network, we realized a group of neural network controllers that enables a stable walk on uneven terrains.

We evaluate the bipedal gait in the simulation environment on multiple classes of terrain obstacles that include bumps, pits and slopes. The results are based on the commercial simulator Webots that uses the Open Dynamics Engine (ODE) for physics simulation. It is possible to make the terrain obstacles arbitrarily difficult and thus we use environments that are challenging while remaining viable. All of the terrains are generated randomly based on Perlin noise and the parameters in Table 4 characterize each type of obstacle.

When the robot walks on the uneven floor and a new step begins with the single support phase, the neural network in the 


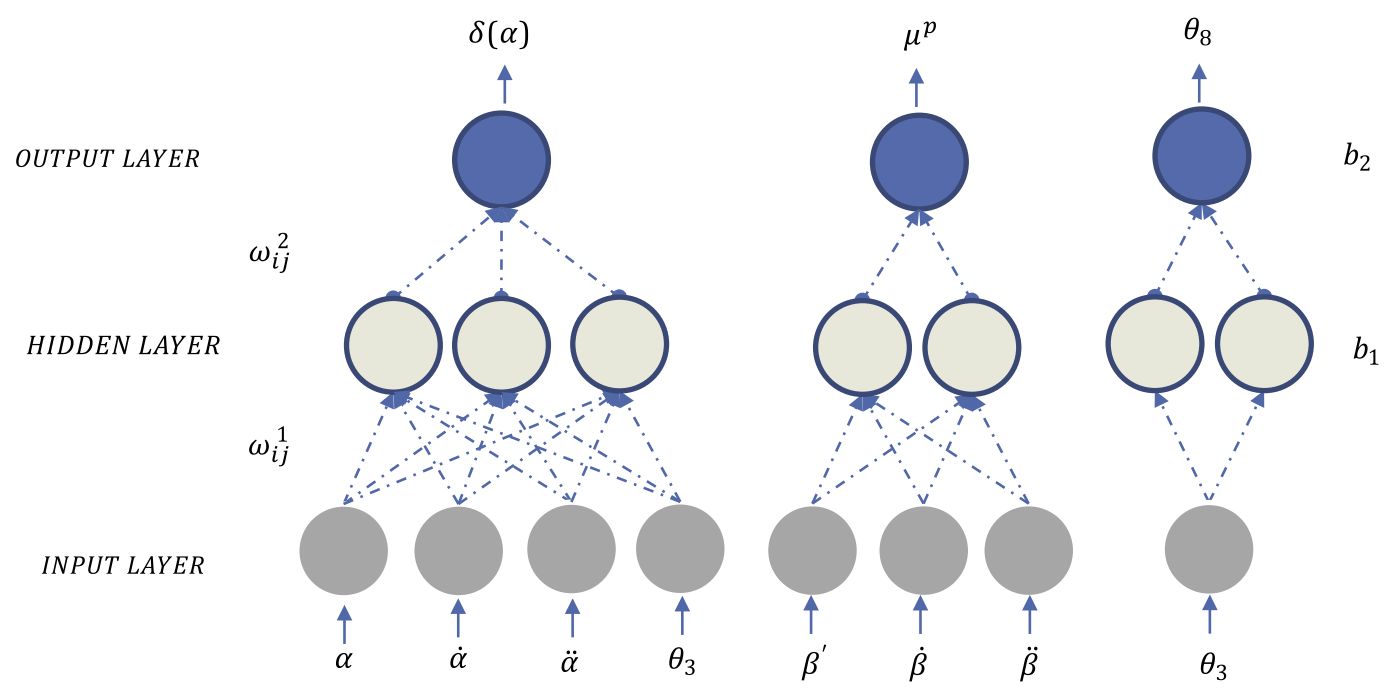

Fig. 10. Backpropagation neural network.

Table 4

The parameters to generate uneven terrain in Webots.

\begin{tabular}{|c|c|c|}
\hline Type & Parameter & Value \\
\hline SFVec3f & Translation & 000 \\
\hline SFRotation & Rotation & 0100 \\
\hline SFVec3f & Size & 50550 \\
\hline SFInt32 & xDimension & 50 \\
\hline SFInt32 & zDimension & 50 \\
\hline SFInt32 & randomSeed & 0.5 \\
\hline SFBool & flatCenter & TRUE \\
\hline SFBool & flatBounds & TRUE \\
\hline SFInt32 & perlinNOctaves & 1 \\
\hline
\end{tabular}

sagittal controller takes the values $\theta_{3}$ (Knee Bending), $(\alpha, \dot{\alpha})$ retrieved from the joint sensors and $\ddot{\alpha}$ from the IMU as the input vector and outputs the leg-length value for the stance leg. When double support phase begins, the first network in the lateral controller uses $\left(\beta^{\prime}, \dot{\beta}, \ddot{\beta}\right)$ as input and outputs the stiffness value of the swing leg, which guarantees that the CoM rotation around ankle stops at the balance point. The second neural network takes the value of $\theta_{3}$ (Knee Bending) as input at the end of the double support phase and outputs the value of $\theta_{8}$, which is used to determine the angle of torso that leans in lateral direction during next double support phase. Together with other fixed parameters, the gait controller generates updated joints commands to avoid CoM undershoot or overshoot caused by uneven terrains and other disturbances.

\subsection{Evaluation of stability}

In order to validate the gait's dynamic stability on randomly generated uneven terrains, we perform a series of experiments with the Nao (version 5) humanoid robot. We implemented the proposed controller and validated the result by letting the robot walk a constant distance ( 0.5 meters). The step size was fixed to $3.9 \mathrm{~cm}$.

Fig. 11 shows the vertical projection of the CoM on the ground. Each foot shown in this figure does not represent an area of support. Because of the low stiffness on the support ankle joint, the ankle can move freely in sagittal and lateral direction. Therefore, the area of support of a foot is constrained to a small area formed by the location of the ankle joint, instead of the foot area that contacts the ground. Each red dot in Fig. 11 indicates the position of ankle joint in the contact area of a foot during one step.
Fig. 11 shows that there is no stability in the sagittal direction of the proposed gait. Stability criteria such as the ZMP are not applicable. Moreover, the figure shows that, in the lateral direction, the CoM trajectory does not overshoot or undershoot the support points (red dots) on uneven grounds, which proves that the proposed gait is stable on randomly generated terrains.

Fig. 12 shows the roll angles trajectories of left and right leg when robot walks on slope in $0.02 \mathrm{rad}\left(\approx 1^{\circ}\right), 0.05 \mathrm{rad}\left(\approx 3^{\circ}\right)$ and $0.09 \mathrm{rad}\left(\approx 5^{\circ}\right)$. In this figure, we can see that left foot is higher than the right one on the slope because the joint of right leg rotates less than the joint on left leg to let the CoM approach its balance point. Moreover, under the different slope angles, the duration of one step does not change, which means that our proposed gait can make a stable walk on different slopes without the loss of walking velocity. The reason why we constrain the slope angle within $0.09\left(\approx 5^{\circ}\right)$ is because the behavior of the robot becomes unstable when it walks on slopes up to $10^{\circ}$ or more. We observed that the chance of a collision between the front of the Naos big feet and slope surface increases as the slope angle increases. The collision undermines the dynamics of the gait and may cause the robot tip over. To avoid this situation, the walking pattern of swing leg needs to be changed and the robot should be able to observe in some way the angle of the surface. For example, given information of terrain, the foot sole can be pre-paralleled with slope to avoid a collision before landing on the ground. This solution is problematic because the slope angle may change and visual feedback is a challenging problem. Another problem is the Nao's smooth sole, which do not provide sufficient grip when the slope angle becomes too large. So, after many tests, we choose $5^{\circ}$ as the maximal slope angle in the demo to ensure the success rate of uphill walking.

The accompanying video material ${ }^{3}$ shows the Nao robot walking on an uneven terrain with our proposed gait controller in the simulator Webots, which shows that our controller can handle the altitude differences of the foot placement and adjusts the control parameters to maintain balance.

\subsection{Evaluation of energy efficiency}

To evaluate the energy efficiency of the proposed gait, we ran trials with the Nao humanoid robot in the simulator environment. In the first experiment, the average electric currents that flow

\footnotetext{
${ }^{3}$ https://www.youtube.com/watch?v=7DxHVEd8hc8.
} 
The CoM Trajectory of Proposed Gait on Uneven Terrain

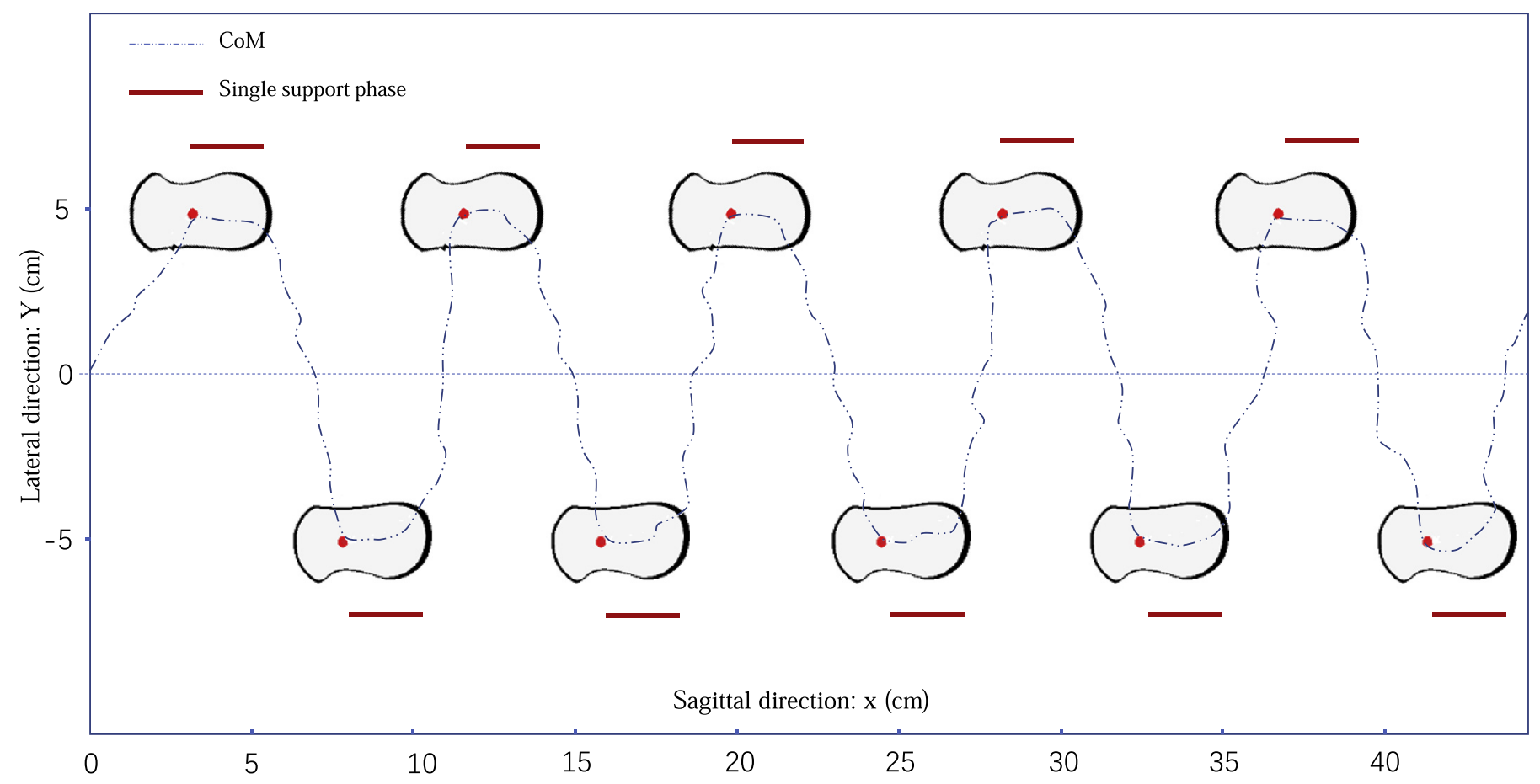

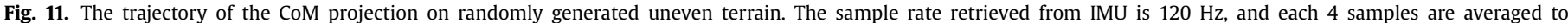

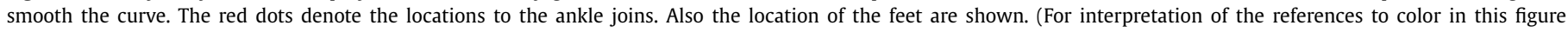
legend, the reader is referred to the web version of this article.)

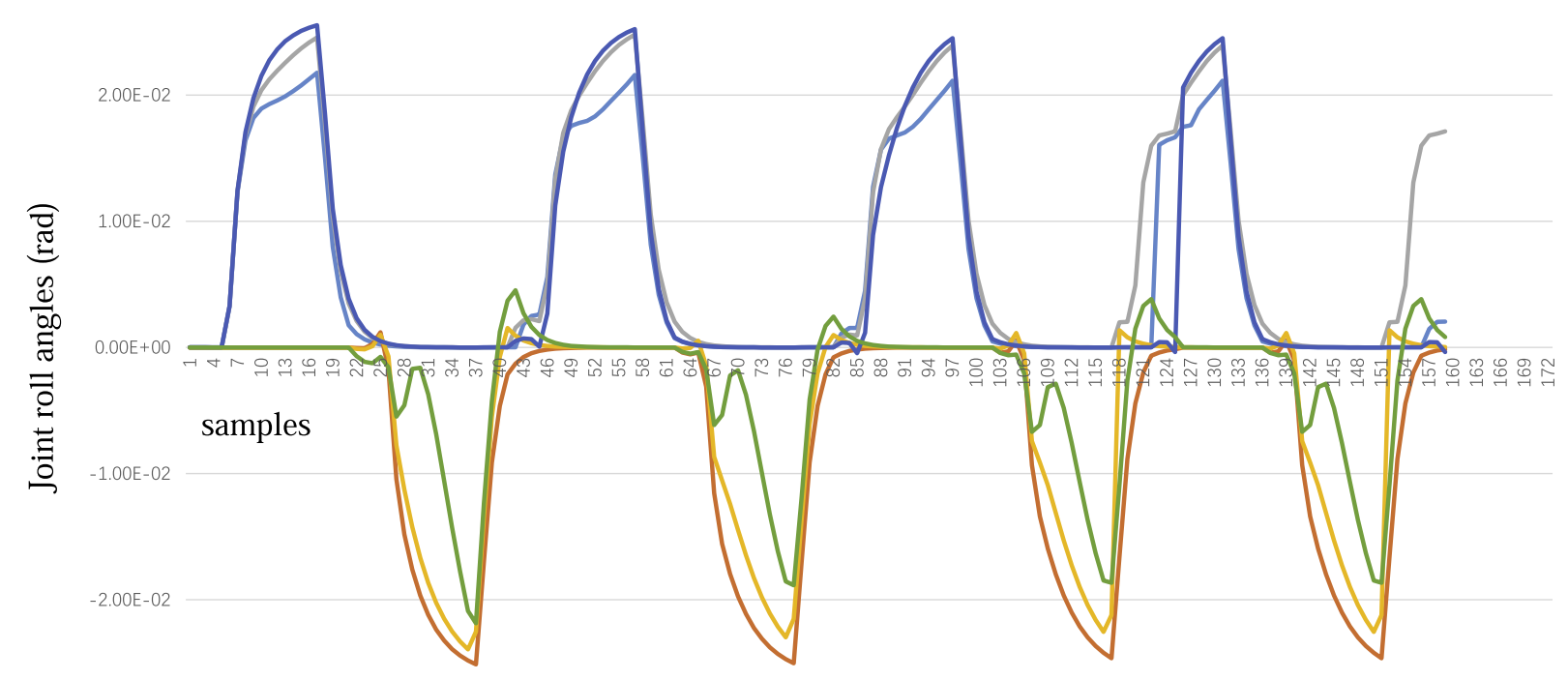

$-3.00 \mathrm{E}-02$

$$
\text { —left1 —right1 —left3 - right3 - left5 -right5 }
$$

Fig. 12. The roll angle trajectory of both legs under different slope in 1, 3, 5 degree.

through the leg joints, are recorded and compared for two gaits: the Aldebaran gait and our new gait, while walking a constant distance on the flat ground. The electric current in the joints for sagittal movement (HipPitch, KneePitch and AnklePitch) and lateral movement (HipRoll and AnkleRoll) are recorded separately. Fig. 13 shows how much the sagittal motion and lateral motion contribute to the total energy consumption. In the hip joints, the average cur- rents for lateral motion (HipRoll) are almost the same (0.095 A in the Aldebaran gait, $0.09 \mathrm{~A}$ in the proposed new gait), but average currents $(0.07 \mathrm{~A})$ in the HipPitch for the sagittal motion in the proposed new gait is $17.6 \%$ less than that in the Aldebaran gait $(0.085 \mathrm{~A})$. The reason is that, compared to Aldebaran gait, we use a larger and proper step size in the proposed gait, which makes the robot cover the same distance in fewer steps. In the knee joint, the 
Electric current consumption on hip, knee, ankle joints of two gaits

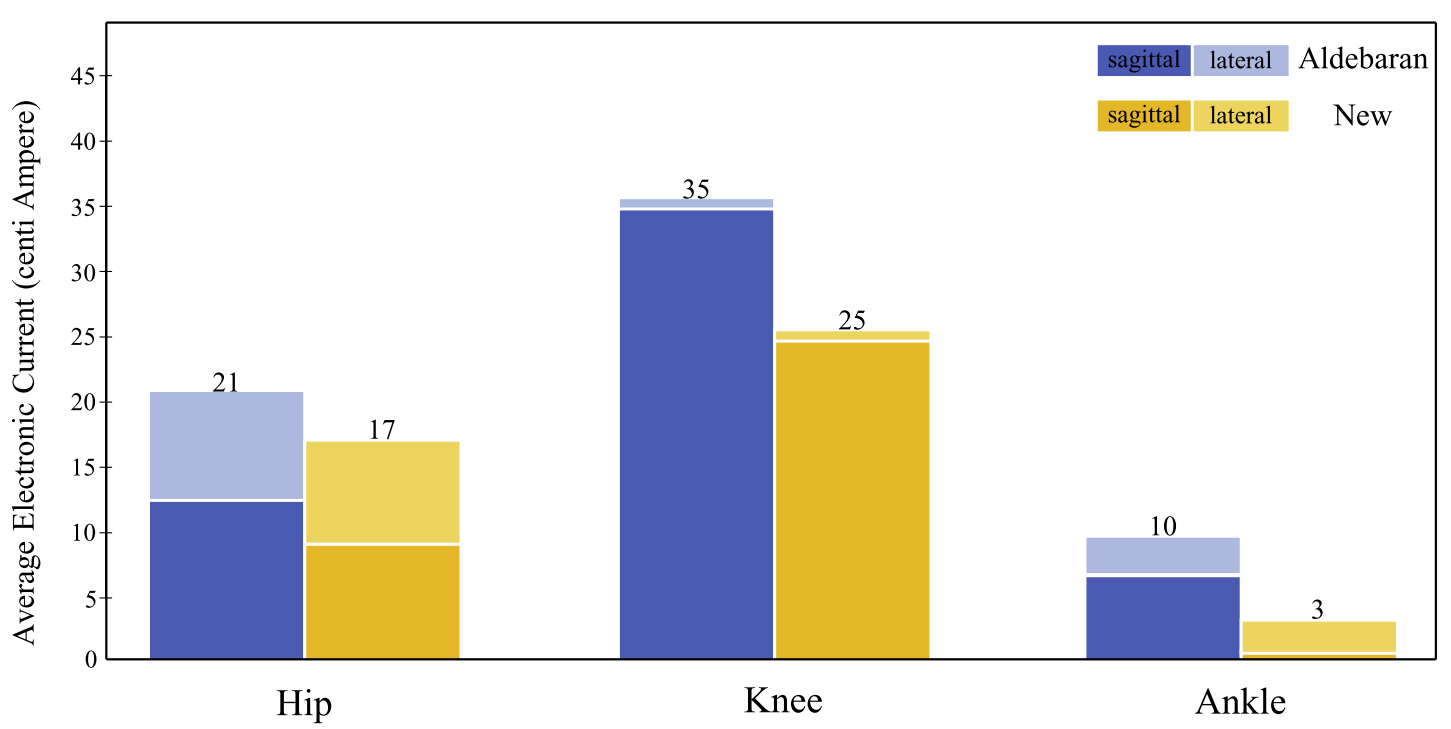

Fig. 13. The electric current consumption on hip, knee, ankle joints of proposed new gait and Aldebaran gait.

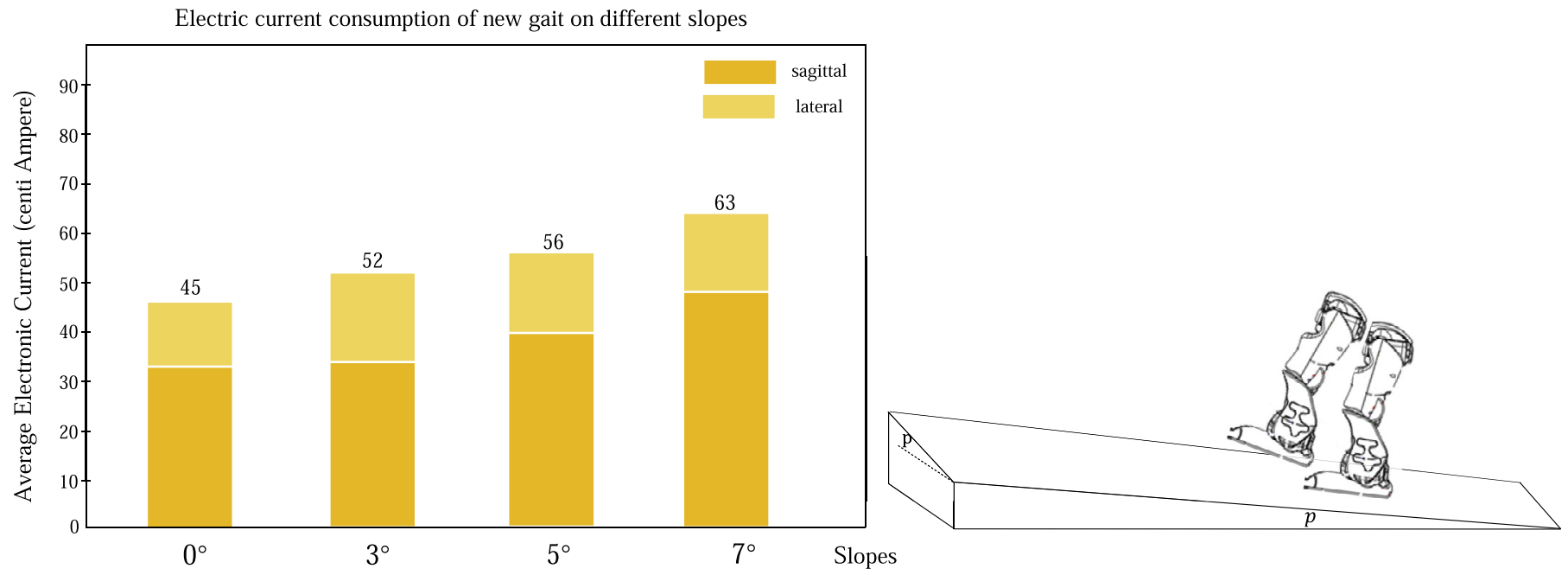

Fig. 14. The electric current of the proposed walk gait on different slopes.

average current $(0.25 \mathrm{~A})$ in the proposed gait is $28.6 \%$ less than the one in the Aldebaran gait $(0.35 \mathrm{~A})$. Regulated by the optimal leg-length policy, the proposed gait keeps the stance leg stretched most of the step time, which has the advantage in energy efficiency over Aldebaran gait. Since the knee joint has only one degree of freedom (KneePitch) in forward direction, it does not have lateral motion thus no lateral energy cost. Moreover, in the proposed gait, the design of a none-stiff ankle joint (AnklePitch) decreases the energy cost for sagittal movement to zero. The average current in a fully stiff ankle joint in the Aldebaran gait is $0.03 \mathrm{~A}$. In the lateral plane, the average current $(0.05 \mathrm{~A})$ in the ankle joint in the proposed gait is $28.6 \%$ less than that in the Aldebaran gait $(0.07 \mathrm{~A})$ because of a low stiffness $(10 \%)$ is applied in the previous one. The overall energy consumption of joints on the leg in our new gait reduces by $31 \%$ compared to the Aldebaran gait on the flat ground. In our previous work [1], we implemented the new gait on a real Nao robot and conducted the same experiment. The results show that, compared to the Aldebaran gait of the Nao on the flat floor, the overall energy consumption of the gait reduces by $41 \%$. The disparity between the energy costs in the simulator and the real Nao robot is caused by the different ways of energy measurement on both platforms. For example, on the real Nao robot, the electric current of joints can be read directly from the joint sensor. Whereas, in the simulation environment, the energy consumption is estimated using the torque applied on the joints.

The second experiment shows the energy consumption of the proposed gait w.r.t the increasing slope angles up to $5^{\circ}$ in the sagittal and lateral plane. The energy costs of the proposed gait are recorded and plotted in Fig. 14. In the experiment, the Nao robot is placed on a slope of angle $p$ both in sagittal and lateral plane. In this way, the energy cost of sagittal motion and lateral motion can be recorded at the same time. We let the robot walk a constant distance on the slope of 0, 3, 5 and 7 degree separately, and record the average electric currents in the joints for sagittal movement (HipPitch, KneePitch, AnklePitch) and lateral movement (HipRoll, AnkleRoll). Fig. 14 shows that more energy is consumed in the joints for the sagittal and the lateral motion as the angle of slope increases. Notably, our new gait on the slopes requires less energy than the Aldebaran gait on the flat ground. This indicates that the proposed gait has better performance not only w.r.t. the stability but also w.r.t. the energy efficiency on uneven terrains. 


\section{Conclusion}

In our previous work we have presented a new gait for humanoid robots. An implementation of the gait on a Nao robot reduces the energy consumption by $41 \%$ compared to the Aldebaran gait of the Nao on a flat floor. An important feature of new the gait is that it does not use an area of support. That is, the robot can rotate freely around the ankle joint while walking. This makes the new gait suited for uneven terrains because the feet can adapt to the slope of the terrain.

The absence of an area of support implies that, in principle, the robot is unstable. In the sagittal plane, the robot falls forwards in each step, and in the lateral plane, the robot balances above the stance foot in the single support phase and falls towards the swing foot in the double support phase. Nevertheless, experiments with a Nao robot on an almost flat floor consisting of wooden planks showed that the gait is stable.

In this paper, we investigate how we can improve the sagittal and lateral stability of the gait when a Nao robot walks on an uneven terrain. We first assume that single step on an uneven terrain can be modelled as a step on the (virtual) slope with a certain angle. This assumption maps various terrain classes to slopes, thereby, transforming the balance control problems on uneven terrain as those on slopes. Since in the single support phase, the robot can turn freely around the ankle joint, a bump or a hole on the walking surface may disrupt the sagittal stability. Moreover, during the double support phase the robot may overshoot or undershoot its stable end point, namely, balancing stable above the new stance foot. We presents a feedback controller based on three feedforward neural networks that adapts the gait parameters in order ensure the robot's stability while it walks on an uneven terrain.

The presented approach shows how to develop simple neural network controllers for an energy efficient gait that enables walking on an uneven terrain:

1. Analyze the gait using an abstract mathematical model of a robot,

2. Build a controller with 8 control parameters implementing the gait,

3. Identify for each slope angle the optimal control parameters using reinforcement learning. The control parameters define a different controller for each slope angle.

4. Use the set of controllers (one for each slope angle) to train a neural network controller that determines the length of the stance leg, and another neural network controller which determines the stiffness of the knee joint of the swing leg in the double support phase.

The method is not limited to the Nao robot and can be applied to humanoid robots with a similar design as a Nao robot, such as KHR-3, DARWIN-OP2. Moreover, the method that we used to develop a new gait can be used for a wider range of robot designs, and even robots with point feet. The only essential sensor in our gait controller is the IMU, which is present in most of the modern robot. The method can also be applied to other movements such as: kicking a ball, running, dancing, walking with foot placement control, etc. Further research into these possibilities is required. In the longer run, the method might be used to analyze the effects of walking disabilities in humans and identify the best way of dealing with these disabilities. Robots can be used to evaluate possible improvements.

\section{References}

[1] Z. Sun, N. Roos, An energy efficient dynamic gait for a NAO robot, in: Proceedings of IEEE Conference on Autonomous Robot Systems and Competitions, 2014.
[2] Z. Sun, N. Roos, An energy efficient gait for a NAO robot, in: Proceedings of Benelux Conference on Artificial Intelligence, BNAIC, 2013.

[3] Z. Sun, N. Roos, Dynamic lateral stability for an energy efficient gait, in: Proceedings of Benelux Conference on Artificial Intelligence, BNAIC, 2014

[4] G.F. Franklin, J.D. Powell, A. Emami-Naeini, Feedback Control of Dynamics Systems, Addison-Wesley, Reading, MA, 1994.

[5] S. Kajita, F. Kanehiro, K. Kaneko, K. Yokoi, H. Hirukawa, The 3D linear inverted pendulum mode: a simple modeling for a biped walking pattern generation, in: Proceedings of 2001 IEEE/RSJ International Conference on Intelligent Robots and Systems, vol. 1, IEEE, 2001, pp. 239-246.

[6] M. Vukobratovic, B. Borovac, D. Surla, D. Stokic, Biped Locomotion: Dynamics, Stability, Control and Application, vol. 7, Springer Science \& Business Media, 2012.

[7] S. Kajita, F. Kanehiro, K. Kaneko, K. Fujiwara, K. Harada, K. Yokoi, H. Hirukawa, Biped walking pattern generation by using preview control of zero-moment point, in: Proceedings of IEEE International Conference on Robotics and Automation, 2003. ICRA'03., vol. 2, IEEE, 2003, pp. 1620-1626.

[8] S. Kajita, K. Kaneko, F. Kaneiro, K. Harada, M. Morisawa, S. Nakaoka, K. Miura, K. Fujiwara, E.S. Neo, I. Hara, et al., Cybernetic human HRP-4c: a humanoid robot with human-like proportions, in: Robotics Research, Springer, 2011, pp. 301-314.

[9] F. Kanehiro, H. Hirukawa, S. Kajita, Openhrp: Open architecture humanoid robotics platform, Int. J. Robot. Res. 23 (2) (2004) 155-165.

[10] Y. Sakagami, R. Watanabe, C. Aoyama, S. Matsunaga, N. Higaki, K. Fujimura, The intelligent ASIMO: system overview and integration, in: Proceedings of IEEE/RSJ International Conference on Intelligent Robots and Systems, 2002, vol. 3, IEEE, 2002, pp. 2478-2483.

[11] T. McGeer, Passive dynamic walking, Int. J. Robot. Res. 9 (2) (1990) 62-82.

[12] A.D. Kuo, Choosing your steps carefully, Robot. Autom. Mag. IEEE 14 (2) (2007) $18-29$.

[13] S.H. Collins, A. Ruina, A bipedal walking robot with efficient and human-like gait, in: Proceedings of the 2005 IEEE International Conference on Robotics and Automation, 2005, IEEE, 2005, pp. 1983-1988.

[14] K. Farrell, C. Chevallereau, E. Westervelt, Energetic effects of adding springs at the passive ankles of a walking biped robot, in: Proceedings of IEEE International Conference on Robotics and Automation, 2007, IEEE, 2007, pp. 3591-3596.

[15] T. Yang, E. Westervelt, J.P. Schmiedeler, R. Bockbrader, Design and control of a planar bipedal robot ERNIE with parallel knee compliance, Auton. Robots 25 (4) (2008) 317-330.

[16] J. Pratt, C.-M. Chew, A. Torres, P. Dilworth, G. Pratt, Virtual model control: an intuitive approach for bipedal locomotion, Int. J. Robot. Res. 20 (2) (2001) 129-143.

[17] C. Chevallereau, Y. Aoustin, Optimal reference trajectories for walking and running of a biped robot, Robotica 19 (05) (2001) 557-569.

[18] T. Saidouni, G. Bessonnet, Generating globally optimised sagittal gait cycles of a biped robot, Robotica 21 (02) (2003) 199-210.

[19] B. Gökçe, H.L. Akin, Parameter optimization of a signal-based omni-directional biped locomotion using evolutionary strategies, in: RoboCup 2010: Robot Soccer World Cup XIV, Springer, 2011, pp. 362-373.

[20] A. Abdolmaleki, N. Shafii, L.P. Reis, N. Lau, J. Peters, G. Neumann, Omnidirectional walking with a compliant inverted pendulum model, in: Advances in Artificial Intelligence-IBERAMIA 2014, Springer, 2014, pp. 481-493.

[21] H. Shahbazi, K. Jamshidi, A.H. Monadjemi, H. Eslami, Biologically inspired layered learning in humanoid robots, Knowl.-Based Syst. 57 (2014) 827.

[22] M. Ogino, H. Toyama, M. Asada, Stabilizing biped walking on rough terrain based on the compliance control, in: Proceedings of IEEE/RSJ International Conference on Intelligent Robots and Systems, 2007. IROS 2007, IEEE, 2007, pp. 4047-4052

[23] J.H. Park, E.S. Kim, Foot and body control of biped robots to walk on irregularly protruded uneven surfaces, IEEE Trans. Syst. Man Cybern. Part B (Cybern.) 39 (1) (2009) 289-297.

[24] S. Sano, M. Yamada, N. Uchiyama, S. Takagi, Point-contact type foot with springs and posture control for biped walking on rough terrain, in: Proceedings of the 10th IEEE International Workshop on Advanced Motion Control, 2008. AMC'08, IEEE, 2008, pp. 480-485

[25] H.-j. Kang, K. Hashimoto, H. Kondo, K. Hattori, K. Nishikawa, Y. Hama, H.-o. Lim, A. Takanishi, K. Suga, K. Kato, Realization of biped walking on uneven terrain by new foot mechanism capable of detecting ground surface, in: Proceedings of 10th IEEE International Conference on IEEE International Workshop on Robotics and Automation (ICRA), 2010, IEEE, 2010, pp. 5167-5172.

[26] L. Vladareanu, G. Tont, I. Ion, M.S. Munteanu, D. Mitroi, Walking robots dynamic control systems on an uneven terrain, Adv. Electr. Comput. Eng. 10 (2) (2010) 145-152.

[27] I.R. Manchester, U. Mettin, F. Iida, R. Tedrake, Stable dynamic walking over uneven terrain, Int. J. Robot. Res. 30 (3) (2011) 265-279.

[28] S.-J. Yi, B.-T. Zhang, D.D. Lee, Online learning of uneven terrain for humanoid bipedal walking., in: Proceedings of Conference on Artificial Intelligence AAAI, vol. 10, 2010, pp. 1639-1644.

[29] K. Sabe, M. Fukuchi, J.-S. Gutmann, T. Ohashi, K. Kawamoto, T. Yoshigahara, Obstacle avoidance and path planning for humanoid robots using stereo vision, in: Proceedings of IEEE International Conference on Robotics and Automation, 2004. ICRA'04. 2004, vol. 1, IEEE, 2004, pp. 592-597.

[30] P. Michel, J. Chestnutt, S. Kagami, K. Nishiwaki, J. Kuffner, T. Kanade, Online environment reconstruction for biped navigation, in: Proceedings of IEEE 
International Conference on Robotics and Automation, 2004. ICRA'04. 2004, IEEE, 2006, pp. 3089-3094.

[31] P. Michel, J. Chestnutt, S. Kagami, K. Nishiwaki, J. Kuffner, T. Kanade, Gpu-accelerated real-time 3D tracking for humanoid locomotion and stair climbing, in: Intelligent Robots and Systems, 2007. IROS 2007. IEEE/RSJ International Conference on, IEEE, 2007, pp. 463-469.

[32] Q. Wang, Y. Huang, J. Zhu, B. Chen, L. Wang, Dynamic walking on uneven terrains with passivity-based bipedal robots, Inform. Control Autom. Robot. (2011) 187-199.

[33] Y. Sugahara, Y. Mikuriya, K. Hashimoto, T. Hosobata, H. Sunazuka, M. Kawase, H.-o. Lim, A. Takanishi, Walking control method of biped locomotors on inclined plane, in: Proceedings of IEEE International Conference on Robotics and Automation, 2005. ICRA 2005, IEEE, 2005, pp. 1977-1982.

[34] O.C. Jenkins, P. Wrotek, M. McGuire, Dynamic humanoid balance through inertial control, in: Proceedings of the 7th IEEE-RAS International Conference on Humanoid Robots, 2007, IEEE, 2007, pp. 501-506.

[35] J.-Y. Kim, I.-W. Park, J.-H. Oh, Walking control algorithm of biped humanoid robot on uneven and inclined floor, J. Intell. Robot. Syst.. 48 (4) (2007) 457-484.

[36] K. Hashimoto, Y. Sugahara, A. Ohta, H. Sunazuka, C. Tanaka, M. Kawase, H.-o. Lim, A. Takanishi, Realization of stable biped walking on public road with new biped foot system adaptable to uneven terrain, in: Proceedings of the First IEEE/RAS-EMBS International Conference on Biomedical Robotics and Biomechatronics, 2006. BioRob 2006, IEEE, 2006, pp. 226231.

[37] G. Taga, Y. Yamaguchi, H. Shimizu, Self-organized control of bipedal locomotion by neural oscillators in unpredictable environment, Biol. Cybern. 65 (3) (1991) 147-159.

[38] T. Matsubara, J. Morimoto, J. Nakanishi, M.-a. Sato, K. Doya, Learning CPG-based biped locomotion with a policy gradient method, Robot. Auton. Syst. 54 (11) (2006) 911-920.
[39] N. Kohl, P. Stone, Policy gradient reinforcement learning for fast quadrupedal locomotion, in: Proceedings of IEEE International Conference on Robotics and Automation, ICRA'04, vol. 3, IEEE, 2004, pp. 2619-2624.

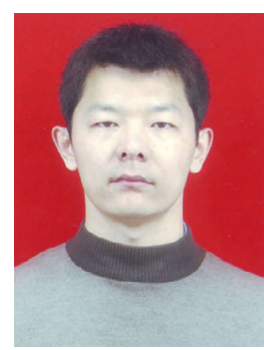

Zhenglong Sun has been working as Ph.D. student with the Department of Data-science and Knowledge Engineering (DKE) of Maastricht University (UM). His research intetersts include bipedal walking control, humanoid whole-body control and motion planning.

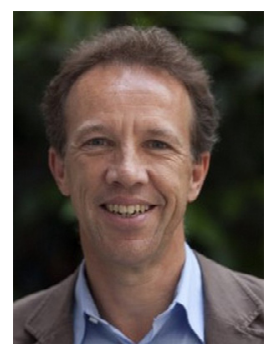

Prof.dr.ing. Nico Roos has a background in electrical engineering and computer science. In 1991, he received his Ph.D. from the Delft University of Technology for his research on the handling of inconsistent and uncertain information. Nico Roos joined the department of Datascience and Knowledge Engineering (DKE) of Maastricht University (UM) in 1995. From the beginning of 2008 till the end of 2010, Nico Roos has been the Director of Studies for the bachelor program Knowledge Engineering, In the same period, he was a member of the Board of DKE. He has also been the chair of the faculty council for 8 year. His current research interests are knowland robotics. edge representation and reasoning, multi-agent systems 